EPJ Web of Conferences 59, 01012 (2013)

DOI: $10.1051 /$ epjconf/20135901012

(C) Owned by the authors, published by EDP Sciences, 2013

\title{
Effect of the laser wavelength: A long story of laser-plasma interaction physics for Inertial Confinement Fusion Teller Medal Lecture
}

\author{
Christine Labaune ${ }^{a}$ \\ LULI, Ecole Polytechnique, CNRS, CEA, UPMC, 91128 Palaiseau, France
}

\begin{abstract}
Laser-driven Inertial Confinement Fusion (ICF) relies on the use of high-energy laser beams to compress and ignite a thermonuclear fuel with the ultimate goal of producing energy. Fusion is the holy grail of energy sources-combining abundant fuel with no greenhouse gas emissions, minimal waste products and a scale that can meet mankind's long-term energy demands. The quality and the efficiency of the coupling of the laser beams with the target are an essential step towards the success of laser fusion. A long-term program on laser-plasma interaction physics has been pursued to understand the propagation and the coupling of laser pulses in plasmas for a wide range of parameters.
\end{abstract}

\section{INTRODUCTION}

Soon after the laser discovery, the idea to build high-power lasers to drive fusion reactions in a small pellet to produce energy was proposed by Jean Robieux [1]. Fusion energy is an attractive, environmentally clean, power source with no greenhouse gases or long-lived radioactive waste materials. An essential step towards ignition is the efficient coupling of the laser beams with the target. During the first picoseconds of the laser pulse irradiating a material, a plasma is produced. The physics of laserplasma interaction includes the propagation of the beams in this plasma and the processes by which the energy of the electromagnetic wave is given to the plasma. Because of the high energy that must be transferred to the target during the laser pulse, laser intensities are such that non-linear processes are expected.

One important parameter for the laser propagation in a plasma is the critical density, which is the maximum electron density in which the electromagnetic wave can propagate. The critical density is inversely proportional to the square of the laser wavelength $\left(\lambda_{0}\right):\left(\mathrm{n}_{\mathrm{c}}\left(\mathrm{cm}^{-3}\right)=1.1 \times 10^{21} / \lambda_{0}(\mu \mathrm{m})^{2}\right)$. The energy deposited in the corona is then transferred by conduction in the denser plasma. The main processes of energy deposition or reflection were identified in the seventies. Among them, it was soon demonstrated that collisional absorption, which transfers the laser energy to the bulk of the plasma, was the only useful mechanism for Inertial Confinement Fusion (ICF). Other laser-plasma coupling mechanisms, also named anomalous absorption processes, were shown to be deleterious for inertial fusion because the energy was given to hot electrons. Suprathermal electrons can preheat the fuel and thus be detrimental to efficient compression.

Among the non-linear processes are the resonant couplings of the incident laser electromagnetic (EM) wave with the plasma modes, which generate scattered electromagnetic waves [2]. So are stimulated Brillouin (SBS) and Raman scattering (SRS), which can be described as the decay of the incident EM wave into a scattered EM wave and an ion-acoustic wave (IAW) or an electron plasma

ae-mail: christine.labaune@polytechnique.fr

This is an Open Access article distributed under the terms of the Creative Commons Attribution License 2.0, which permits unrestricted use, distribution, and reproduction in any medium, provided the original work is properly cited. 
(EPW) wave respectively. These instabilities, which have a maximum growth rate for the scattered wave propagating in the backward direction, reduce the efficiency and symmetry of the energy transfer from the laser to the target. It is important to notice that these decays are resonant, following the conservation laws $\omega_{0}=\omega_{1}+\omega_{2}$ and $\mathrm{k}_{0}=\mathrm{k}_{1}+\mathrm{k}_{2}$ where $\omega_{\mathrm{i}}$ and $\mathrm{k}_{\mathrm{i}}$ are the frequency and wavenumber of the incident and daugther waves. With stimulated backscattering instabilities, filamentation and self-focusing of the laser beam are the most threatening interaction processes expected in ICF targets.

Modelling these non-linear mechanisms is not easy, especially because they mix very different spatial and temporal scales. The situation ended being very complex as not only the daughter waves associated with the instabilities were observed to interact with each other but could also decay into secondary modes and produce cascades of waves. The situation seemed desperately complex and a strategy was needed to control interaction physics in ICF. A long-term program was set up associating the experimental, theoretical and numerical approaches. An important step was the design of wellcontrolled experiments in well-characterized plasmas with powerful diagnostics. The possibility to use multiple laser beams was essential to separate the plasma production from the interaction beam and to use probe beams for diagnostics, in particular Thomson scattering.

The strategy, which was implemented by Edouard Fabre at the LULI (previously PMI) laboratory at the end of the sixties, was to set up very versatile laser facilities to study the physics involved in the field of laser-produced plasmas for ICF but also for all possible applications. He also had the vision of anticipating the laser evolution to follow the progress in the field, like shortening the laser wavelength and going to short pulses. Thus different lasers were installed in the laboratory from the ruby laser, $\mathrm{CO}_{2}$, and neodymium laser in its fundamental wavelength and its harmonics. He ran the program demonstrating that high conversion efficiencies of the fundamental frequency of the neodymium laser could be obtained with non linear crystals following the studies on non linear optics of $\mathrm{N}$. Bloembergen in 1962. With the available energies of these lasers, the idea was to study fundamental physics that could apply to conditions relevant to fusion experiments. It was necessary to find target and beam configurations that could emulate plasma conditions interesting for the different studies. Several kinds of targets have been studied for interaction physics, from thick and thin solid targets, to gas jets and foams, using multiple beam irradiations to produce and heat the plasma independently of the interaction beam(s). For certain studies, it was faster to use outside facilities and French and international collaborations have been established, in particular with the Commissariat à l'Energie Atomique (CEA) to conduct experiments with, in 1990, the highest power/short pulse laser, P102 and later on with the highest laser energy in Europe, the LIL facility.

The issues of laser-plasma interaction in the context of laser fusion depend on the scheme: direct/indirect-drive [3] and new ignition schemes. For all cases, efficient energy absorption is required. In the case of direct-drive, the laser beams propagate in a long and hot plasma, the corona, which has an electron density growing from outside to inside the target. The main objectives are to avoid instabilities that produce i) irradiation inhomogeneities that can seed hydrodynamics instabilities, like filamentation and self-focusing, ii) losses of energy or modification of the symmetry of irradiation, like SBS and SRS, iii) suprathermal electrons that can preheat the core, like SRS and the two-plasmon instability. In the case of indirect-drive, there are mainly three particular zones where parametric instabilities can grow, spoiling the coupling efficiency and symmetry of irradiation: i) the entrance hole, ii) the long plasma inside the cavity, and iii) the hohlraum wall. The main concerns are losses of energy through SBS and SRS, energy transfer between crossing beams through forward SBS, nonresonant reamplification of backscattered light, hot electron generation by SRS and beam bending due to flow velocity. Even if the conditions of interaction are different, a lot of physics on parametric instabilities can be shared between the two schemes like the thresholds, growth rates, saturation processes, crossing beam effects, coupling between instabilities, influence of plasma parameters and damping of the waves.

New ignition schemes have renewed the need to control laser-plasma interaction physics. In particular, the shock ignition scheme [4] relies on the capability of the shock pulse to propagate and deposit its energy in the plasma corona of the compressed target. At the high intensities that are required 


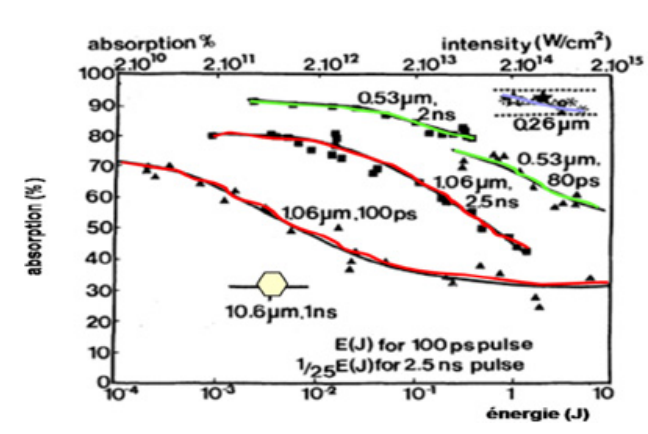

Figure 1. Absorption rates as a function of the laser intensity for different laser wavelength for long $(2 \mathrm{~ns})$ and short (100 ps) pulses.

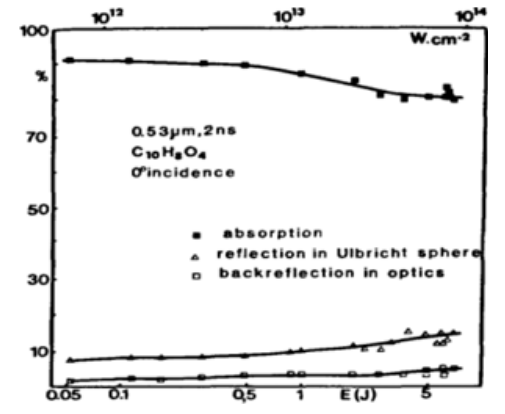

Figure 2. Detailed optical energy balance at $0.53 \mu \mathrm{m}$ for the long pulse regime.

to generate a high shock pressure ( $\sim 400$ Mbars), most of the laser-plasma instabilities (LPI) will be above threshold. Among the risks that can be foreseen are: i) self-focusing and filamentation of the shock laser beam and ii) stimulated Brillouin and Raman scattering that may reduce the efficiency of the coupling and consequently the shock pressure. The hole-boring scheme relies on the possibility of a short and intense laser pulse to dig a hole in the plasma and to transfer its energy to high-energy particles that can deposit their energy in the core of the target. These types of studies build a bridge towards high-intensity studies.

In this paper, we present the historical development of the results of laser-plasma interaction physics that may contribute to determining the laser wavelength of future laser-fusion reactors.

\section{INITIAL ABSORPTION AND LPI MEASUREMENTS}

The choice of an optimum laser wavelength for laser fusion remains an outstanding issue. From the point of view of laser-plasma coupling, it was predicted theoretically and verified experimentally in the 80's that short laser wavelength improved laser beam coupling to the target due to increased collisional absorption (also called Inverse Bremsstrahlung, I.B.) and reduced parametric instabilities [5]. Total absorption as a function of the laser intensity is shown in Fig. 1 for three wavelengths of the neodymium laser $(1.06,0.53$, and $0.26 \mu \mathrm{m}$, also called first, second and fourth harmonics) using short and long pulses, and for the $\mathrm{CO}_{2}$ laser $(10.6 \mu \mathrm{m})$. These measurements were obtained for plastic foils at normal incidence using large aperture focusing optics and an Ulbricht sphere. The data show larger absorption fractions for shorter laser wavelength, lower laser intensities and longer pulse lengths. These results follow exactly what was expected from the scaling laws of collisional absorption. The larger IB absorption at short wavelength comes from the increased density that can be reached by the laser resulting in higher electron-ion collision frequency in the absorption region. Another very interesting result was the low level of SBS backscatter at $0.53 \mu \mathrm{m}$ for long pulses $(2 \mathrm{~ns})$ up to $4 \times 10^{14} \mathrm{~W} / \mathrm{cm}^{2}$. The SBS reflectivity, together with the total reflectivity is shown as a function of the laser intensity for a pulse duration of $2 \mathrm{~ns}$ in Fig. 2. SBS backward reflectivity stays below $5 \%$ of the incident energy.

In addition to a better collisional absorption, the threshold for parametric instabilities increases with shorter laser wavelength. For laser-plasma coupling, the shortest laser wavelength was identified as the best. But a wavelength of $0.26 \mu \mathrm{m}$ was shown to produce too much optical damage. Following these studies, the Laser MégaJoule (LMJ) [6] and National Ignition Facility (NIF) [7] were designed in the early 90's, to reach ignition of an inertial confinement fusion (ICF) capsule in the laboratory with $0.351 \mu \mathrm{m}$ light, the third harmonic $(3 \omega)$ of Nd-glass laser. However, in terms of the construction and operation of the laser, the $0.53 \mu \mathrm{m}$ light could significantly enhance the capabilities and reduce the cost of big lasers. Some advantages of the $2 \omega$ light compared to $3 \omega$ include higher damage threshold 
for the optics [8], more available laser energy and greater achievable bandwidth, allowing enhanced laser smoothing. This last effect was not included in the measurements of the 80's experiments and consequently not in the conclusions.

\section{LASER INCOHERENCE}

Beam smoothing techniques were introduced in 1984 [9] to improve irradiation uniformity and to suppress hydrodynamic instabilities. It has been observed that laser beam smoothing had the potential to control the scattering level from parametric instabilities since they provided a reduced coherence. The laser coherence directly affects the growth of parametric instabilities through the resonant relations, presented in the introduction, that get detuned. Beam smoothing techniques include spatial smoothing and temporal smoothing by laser bandwidth. Spatial smoothing modifies the phase front and spatial distribution in the focal volume $(\delta \mathrm{k})$. It breaks the beam up into fine-scale structures. Temporal smoothing rapidly varies the fine-scale structure. It reduces the coherence time $(\delta \omega)$ with time and consequently limits the growth time of the instabilities.

Many experiments, with different plasma conditions, have demonstrated the reduction of SBS, SRS and filamentation with spatial and temporal smoothing. Among the most recent ones is the observation of a reduction by factors 2.5 and 2 of the backward SBS and SRS respectively in LIL shots using the $14 \mathrm{GHz}$ modulator compared to shots with the $2 \mathrm{GHz}$ modulator [10]. These measurements were done with $7 \mathrm{mg} / \mathrm{cc}$ TAC foams of $500 \mu \mathrm{m}$ length. These LIL results show for the first time that the longitudinal SSD also reduces stimulated Brillouin and Raman scattering, which is important for the future experiments with the Laser Megajoule.

In addition to the previous techniques, polarization smoothing (PS) consists in dividing the light power into two crossed polarizations with slightly different angular directions [11]. This technique reduces the intensity contrast of the focal spot pattern and the number of high-intensity speckles, which are the ones that contribute the most to parametric instabilities. Clear reduction of SBS, SRS and filamentation was also observed with PS.

However, at that time, no good agreement between numerical simulations and experimental results could be obtained on the effect of laser smoothing on LPI because an important plasma effect was not correctly described.

\section{PLASMA (OR SELF-INDUCED) SMOOOTHING}

This effect was first observed from the temporal evolution of the intensity distribution of the transmitted light of a RPP laser beam after propagation through a preformed plasma [12]. This was interpreted as due to the so-called "plasma-induced incoherence" (PII), which results from the ability of an underdense plasma to induce additional spatial and temporal incoherence [13] to an incident spatially randomized laser beam during its nonlinear propagation. The plasma smoothing process comes from the nonlinear coupling between stimulated Brillouin forward scattering and the dynamically evolving hot spots of the beam. Experiments have observed signatures of PII like the angular and spectral broadening of the forward scattered light, which corresponds respectively to an increased spatial and temporal incoherence.

Quantitative measurements of the reduction of the coherence time and of the degree of spatial coherence of a laser after propagation through a well-defined plasma have been obtained with interferometric techniques. They are shown as a function of the laser intensity in Figures 3 and 4 respectively [14]. The plasma was preformed and heated from a thin exploded $\mathrm{CH}$ foil by three $0.53 \mu \mathrm{m}$ beams and the interaction beam was at $1.053 \mu \mathrm{m}$ and smoothed by a random phase plate (RPP). We observe a drastic reduction of both the coherence time and the degree of spatial coherence for the light having propagated through the plasma compared to vacuum. This reduction increases with the laser intensity. 


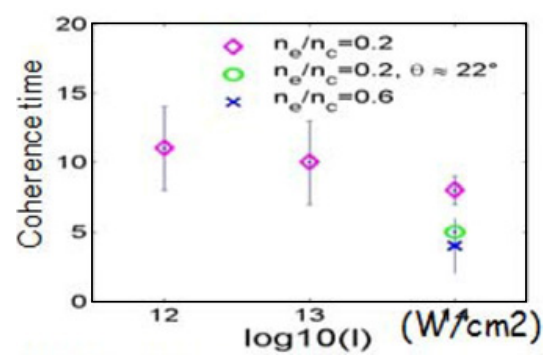

Figure 3. Coherence time of the beam after propagation through a plasma as a function of the laser intensity.

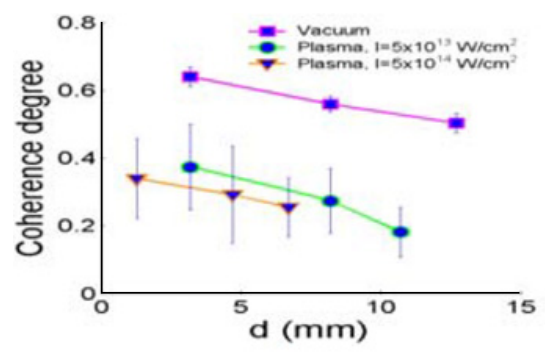

Figure 4. Coherence degree of the beam after propagation through a plasma compared to vacuum.

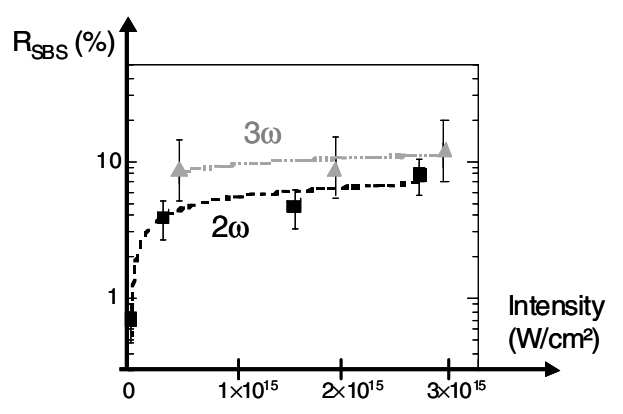

Figure 5. Time-integrated SBS reflectivities at $2 \omega$ and $3 \omega$ as a function of the laser intensity

Using realistic plasma density and expansion velocity profiles, in which the effects of inhomogeneity, non-stationarity, and the light absorption were taken into account, simulations of plasma-induced incoherence reproduced extremely well the LULI experimental results [15]. The improvement on the agreement between numerical simulations and experiments was a major step for interaction physics, demonstrating that we mastered the physics included in the codes. These codes were used to study the impact of PII on stimulated scattering processes, showing that SBS was strongly reduced by PII. A new scheme for direct-drive ICF targets was proposed based on a thin layer of foam to reduce the laser imprint by laser beam smoothing in the foam plasma before the absorption zone which demonstrated a homogeneous ablation pressure during the time of foam decay [16].

\section{SATURATION OF THE INSTABILITIES: COMPARISON OF $2 \omega$ VERSUS $3 \omega$ LIGHT}

One strategy in laser-fusion experiments is to keep the laser intensity below threshold for most of the parametric instabilities [17]. However, a few critical regions exist, both in the direct and indirect drive schemes, where the linear gains for parametric instabilities may reach significant values $(>20)$ for some time during the ignition pulse. Non-linear evolution of the instabilities will lead to saturation. Several mechanisms of saturation have been identified, but it is still impossible to predict the level of saturation with the numerical simulations. Experiments have been performed to compare the saturated levels of SBS reflectivity at 0.53 and $0.35 \mu \mathrm{m}$ [18]. Great care was taken to get results in comparable situations and with complete electron density profiles. The SBS reflectivity was observed to saturate at the same level, $\sim 10 \%$, at 0.53 and at $0.35 \mu \mathrm{m}$ irradiation as shown in Fig. 5. The analysis 


\section{EPJ Web of Conferences}

shows that the interplay between SBS and the mechanisms involved in PII is responsible for SBS saturation.

\section{CONCLUSION}

Although many mechanisms are entangled, the interplay between stimulated scattering processes and plasma smoothing mechanisms demonstrates the potential control of parametric instabilities by reducing the spatio-temporal coherence of the incident laser light. The results are supported by other experiments [19] which confirm the limited levels of SBS and SRS with $2 \omega$ light. The $0.53 \mu \mathrm{m}$ option for laser fusion [20] looks very promising. Going to the future, completely incoherent laser light, as proposed in the scheme of multiple-fibre lasers [21] or with the STUD pulses (Spike Train of uneven Duration and Delay) [22] should drastically reduce all parametric instabilities.

The understanding of laser-plasma interaction has increased dramatically during the past decades. We started from a very complex situation where all the nonlinear phenomena looked harmful for laser fusion. Now, we know how to control them to the point of using them in a positive way like it has been demonstrated in recent NIF experiments where the illumination symmetry is controlled through the exchange of energy between beams [23]. Apart from ICF, many applications have arisen from laserplasma interaction physics. For example, laser acceleration of particles originated from the observation of high-energy ions and electrons produced in laser plasmas. They were mainly due to stimulated Raman forward scattering which was a deleterious mechanism in the context of fusion and became the starting point of the laser-particle acceleration program. Similarly, the observed exchanges of energy due to beam crossing in ICF experiments became a new scheme for laser pulse amplification using stimulated Brillouin or Raman scattering in a plasma [24]. This may lead to the production of super high intensity laser pulses. The extension of laser-plasma interaction physics to very high intensities opens a new way to explore fundamental physics with lasers [25].

This text is the following of the Teller Medal lecture at IFSA 2011, but it has been limited to only part of it to respect the six allocated pages. I dedicate this medal to Edouard Fabre, who built the laboratory with an extraordinary scientific vision and made possible the work acknowledged by the award. I cannot mention the great number of colleagues who shared and contributed to these adventures. However, I would like to thank explicitely H.A.Baldis, S. Depierreux, K. Estabrook, S. Hüller, G. Laval, K. Lewis, P.-E. Masson-Laborde, C. Max, A. Michard, R. Mc Crory, P. Michel, G. Mourou, R. Pellat, D. Pesme, W. Rozmus, W. Seka, C. Stenz and V. Tikhonchuck, whose contributions have been so critical to the success of the program. The perfect harmony that prevailed in the group for several decades has led to exceptional working conditions by the meeting of experimentalists, theoreticians and numerical analysts. Finally, I thank the continuous support of Gérard, Anne-Lise and Julien, which had been very important all along these years and still is.

\section{References}

[1] J. Robieux, "High Power Laser Interactions", Lavoisier (1999); Patent n¹.533.156 (1963)

[2] W.L. Kruer, The Physics of Laser Plasma Interactions (Addison Wesley, Reading, MA, 1988); D. Pesme, C. Labaune, "La Fusion Thermonucléaire Inertielle par Laser”, vol. 1, R. Dautray, J.P. Watteau, editors, Eyrolles, Paris (1993)

[3] J. D. Lindl, Phys. Plasmas 2, 3933 (1995)

[4] R. Betti et al., Phys. Rev. Lett . 98155001 (2007)

[5] C. Garban-Labaune et al., Phys. Rev. Lett. 48, 1018 (1982); C. Garban-Labaune et al., Phys. Fluids 28, 2580 (1985)

[6] M. L. André, Fusion Eng. Des. 44, 43 (1999) 
[7] J.A. Paisner, E.M. Campbell and W.J. Hogan, Fusion Technol. 26, 755 (1994)

[8] H. Bercegol et al., Proc. of SPIE, Vol. 5273, 312 (2004)

[9] Y. Kato, et al., Phys. Rev. Lett. 53, 1057 (1984); S. Skupsky et al., J. Appl. Phys. 66, 3456 (1989)

[10] P.-E. Masson-Laborde et al., these proceedings

[11] LLE Review 45, 1 (1990)

[12] C. Labaune et al., Phys. Fluids B4, 2224 (1992)

[13] A. Schmitt and B. Afeyan, Phys. Plasmas. 5, 503 (1998)

[14] J. Fuchs, C. Labaune et al., Phys. Rev. Lett. 88, 195003 (2002); P. Michel, C. Labaune et al., Phys. Rev. Lett. 92, 175001 (2004)

[15] C. Labaune et al., C.R. Acad. Sci. 4, 727 (2000)

[16] S. Depierreux et al., Phys. Rev. Lett. 102, 195005 (2009)

[17] C. Niemann et al., Phys. Rev. Lett. 100, 045002 (2008)

[18] S. Depierreux et al., Phys. Rev. Lett. 103, 115001 (2009)

[19] K. Oades et al., Bull. Am. Phys. 46, 292 (2001); J. Moody, et al., Ibid 47, 289 (2002)

[20] E.M. Campbell et al., Comments Plasma Phys. Cont. Fusion 18, 201 (1997)

[21] C. Labaune et al., Opt. Comm. 281, 4075 (2008)

[22] B. Afeyan, S. Hüller, these proceedings

[23] P. Michel, et al., Phys. Plasmas 17, 056305 (2011)

[24] V.M. Malkin, G. Shvets, and N. Fisch, Phys. Plasmas 7, 2232 (2000)

[25] G. Mourou, et al., Opt. Comm. 285, 5, 720 (2012) 